\title{
Using Mobile Application to Improve Doctor-Patient Interaction in Healthcare Delivery System
}

\author{
Ekwonwune Emmanuel Nwabueze ${ }^{1}$, Onuoha 0ju² \\ ${ }^{1}$ Department of Computer Science, Imo State University, Owerri, Nigeria \\ ${ }^{2}$ Department of Computer Science, Abia State Polytechnic, Aba, Nigeria \\ Email: ekwonwuneemmanuel@yahoo.com
}

How to cite this paper: Nwabueze, E.E. and Oju, O. (2019) Using Mobile Application to Improve Doctor-Patient Interaction in Healthcare Delivery System. E-Health Telecommunication Systems and Networks, 8, 23-34.

https://doi.org/10.4236/etsn.2019.83003

Received: April 12, 2019

Accepted: September 27, 2019

Published: September 30, 2019

Copyright (c) 2019 by author(s) and Scientific Research Publishing Inc. This work is licensed under the Creative Commons Attribution International License (CC BY 4.0).

http://creativecommons.org/licenses/by/4.0/

\begin{abstract}
The use of mobile applications for healthcare has become a focal point of innovation that could aid patients in their everyday healthcare management. Mobile applications can contribute to increasing the effectiveness and reducing the costs of healthcare delivery as well as preventing illnesses through behaviour change. This paper presents model of an interactive mobile healthcare application that facilitates the needs between doctors and patients. The proposed mobile application will provide optimal communication to several parties in the healthcare sector such as patients, doctors and pharmacists for better healthcare services and delivery. The software development methodology adopted was Object Oriented Analysis and Design Methodology (OOADM). Our mobile application is developed for Android platform and HTML, CSS and JS were used at the front-end while Angula JS, PHP and MYSQL powered the back-end.
\end{abstract}

\section{Keywords}

Mobile Healthcare, Interactive, Technology, Application, Communication

\section{Introduction}

Healthcare is an environment that has been experiencing dramatic progress in computing technology in order to process and distribute all relevant patient information electronically and overall to improve the quality of care. In particular, mobile e-health involves a spectrum of information and telecommunication technologies to provide healthcare services to patients who are at some distance from the provider and also provide supporting tools for the mobile healthcare 
professional. The benefits of such applications are numerous with the main one being improvements in access to medical resources and care.

Recently, the healthcare and related sectors have been found to embrace mobile technology in e-healthcare applications. Though there have also been cases of mobile workstations being implemented at small medical units to facilitate easier access to specialist medical advice, most of the applications have been introduced to support patients at home [1]. These could either be patient-centred where patients and/or caretakers are given direct access to a mobile phone for communicating with the provider (e.g. nurse, doctor, counsellor etc.), or nurse-centred where nurses who visit and care patients at home have direct access to mobile applications and for communicating with other medical staff.

Mobile healthcare has huge potential to improve efficiency, improve healthcare quality, enable doctors to monitor their patient's health, enable patients to manage their heath more comfortably out of the hospital, allow home care providers to provide better healthcare quality to seniors, and reduce the cost of care by allowing patients to make fewer visits to their doctor [2]. A new study suggests that patients who read their e-health records are more likely to understand their medical issues better and to take their medications as prescribed [3]. It is believed that e-health applications also make patients feel more in control and less confused, increase devotion to medications [3], improve relationships between doctors and patients, help patients to ask more informed questions and ease anxiety and confusion without extra work for health providers [4]. Additionally, [5] quoted Dr. Jonathan Pell, an assistant professor at the University of Colorado in Denver as saying "The hope is that increased transparency achieved by sharing electronic medical records with patients while they are in the hospital would make them more engaged in their care, more satisfied, and more likely to ask questions and catch errors". However, some experts think that too much information can cause panic for the patient. It is also thought that patients with less education may need more work to explain health issues to them; while educated patients may have an easier time understanding their health records [5].

\section{Statement of the Problem}

In Nigerian culture, doctor-patient interaction is dominated by the doctor and culture of "not questioning the doctor". Due to social and educational barriers, doctors feel there is no point in attempting to explain tests and test results to patients as they would not understand anyway. The level of communication is placed in the hands of the physician where they are the communicator and patients are the listeners. Doctors were once seen as god-like in the society, the popular belief that they can treat and cure any condition. Hence, patients used to leave their full confidence of their health outcome to them. However, over the past decade, doctors are often criticized for not providing adequate considerations to the patients' feeling or desires regarding the illness or conditions being treated. The patients on the other hand, often assume a doctor may know things about his/her condition when he or she actually does not. These are complex 
problems that arise when the communication gap between doctors and patients is wide. Moreover, patients or family members often sign consent forms without really understanding what they are consenting to. Such one-way communication and decision making disempowers patients who, as a result, rarely participate in their own care. Poor communication is linked with less accurate diagnoses, suboptimal patient compliance, over-treatment, under treatment and "mistreatment" [6].

\section{Aim and Objectives of Study}

The aim of this work is to develop a mobile application that provides optimal communication among patients, doctors and other stakeholders for better healthcare services and delivery. It will help them to communicate with each other for appointment, prescription management and patient data management at any time using mobile with the internet. The objectives of the research are:

1) To create an application that will be easy for use without learning any additional skills.

2) To develop an application that focuses on how to enhance users' awareness and knowledge about medication and its management.

3) To create the framework that will enable more citizens to receive healthcare services whenever needed.

4) To provide mobile platform that will deliver services that are cost-effective and meet certain pre-established standards of quality.

\section{Significance of Study}

The major significance of this work is to provide a mobile healthcare app service with the potential to achieve the key attributes of mobile technology:

1) Access: patients, doctors and other stakeholders will access electronic mobile records for viewing and entering real time data anytime and anywhere. It will provide them easy access for real time communications, information sharing, collaboration and consultation.

2) Insight: the app will facilitate the availability of "interpreted" information using clinical documentation, test results, image data and communication for more efficient collaboration between patients and other stakeholders making faster, more accurate judgments and decisions.

3) Convenience: this app when connected is always on, and "in hand", for the task at hand. This convenience reduces time spent "managing" records, improves data capture, reduces errors, and enables health care providers to have higher quality interactions with patients.

\section{Literature Review}

Many Medication Systems have been developed based on different platforms and concepts. The use of healthcare-related apps is growing but there are many issues related to their functionality. In this section, we reviewed some of the 
literatures and applications that are related to our work.

\section{1) Epocrates}

Epocrates enables patient care by delivering the right information, when it is needed. It has features such as; find providers for consults and referrals in the Provider Directory, review drug prescriptions and safety information, check for potentially harmful drug interactions, access timely medical news and research information, disease information, alternative medications, lab guides, and more clinical tools [7].

\section{2) Doctor at Work}

This application manages patient records, appointments, patient visit notes, bill patients, track customer payments and balance due. This app can be useful for medical professionals and students that visit patients every now and then. It also helps the patients to get the appointments with doctors and sends the reminder through SMS or by email, creates itemized bills for patients to track the due amount, maintains the visit history of the patients, etc. [7].

\section{3) Diagnotes}

This mobile application is web-based and provides healthcare practitioners the tools to enhance doctor interaction with their patients and other support staff. The user is expected to first create his account to enable him have access to all the functionalities the application. It routes phone calls and text, supports documentation of every encounter for continuity of patient care (Google Play, 2015).

All the existing applications discussed above are kind of more commercial and money making, but this our proposed system cares more for patient-centered approach, and provides an optimal communication between doctors and patients. This app is helpful to patients to ask questions and state their concerns to doctors regarding their health condition. This app will facilitate the patients to interact with doctors without making any physical appointments.

\section{Proposed System}

The proposed system is a mobile application that can accommodate the communication needs between doctors and patients. The application must be able to handle processes ranging from the doctor's search process, check registration, queue number settings and notifications, easy-to-access medical records, and chats between doctors and patients. This, therefore, helps in making critical information more readily available for review on an individual basis.

These changes will be more than automated and more efficient versions of what we already do. There will be new ways to support and even provide healthcare: replacements and refinements for existing processes, procedures, and work habits that will improve outcomes. Other benefits of the proposed new system are:

\section{- Improved Communication between Patients and Providers}

This mobile application improves healthcare by making readily available instructions for patients. The greater part of what doctors tell patients is forgotten 
by the time they leave the hospital and half of what they remember is incorrect. Better communication can also take place through mobile app before patients enter their physician's office. Mobile technology can be used to send patient reminders and decrease the number of missed appointments, which leads to a reduction in costs.

\section{- Mobile Healthcare Technology Decreases Readmissions}

The patient's healthcare experience does not stop once they leave the hospital. Providers need to find ways to effectively communicate with patients throughout their healthcare journey or face further health problems in the long-term. Today's healthcare providers face a growing number of readmissions, with so many patients coming back to them within a short while of care. When mobile technology is used to empower patients with information on how to better manage their health, the level of readmission decreases resulting in less cost for the patient.

\section{- Attracting New Patients}

The availability of healthcare apps on the internet helps patients quickly access reliable information when they feel sick. This speaks to the growing desire for mobile healthcare content. By offering this content, a healthcare system could gain the attention of a user early in his life, thus increasing the likelihood that he would choose that system for future healthcare needs.

\section{Methods and Design}

The development of any software requires following certain procedures in order to achieve set objective. There are many methodologies that are adopted by software developers to provide pleasant software development based on the project needs. For the software development of the proposed system, Object Oriented Analysis and Design Method (OOADM) were used.

\section{System Requirements}

Hardware Requirements:

- Laptop.

- Android Phone.

Software Requirements:

- Operating System: Android OS; Windows 8.

- Front-End: HTML, CSS, JS and Android studio.

- Back-End: PHP, MYSQL.

The interactive healthcare application architecture consists of three major parts Graphical User Interface (GUI), front end and back end. The architecture displays the basic flow of the system. Through GUI the system will interact with the user. GUI allows the use of icons or other visual indicators to interact with users. It will display the different categories such as patient reports, doctor's prescriptions, doctor and patient details etc. When the patient or doctor clicks on the particular link, the query goes to the front end part. After that front end 
fetches the required data from the database i.e. backend. The results are returned to front end and from there to GUI for displaying as shown in Figure 1. There is a database in the back end which handles all the crucial data regarding patient, doctor, pharmacy, admin etc.

Our application iMobiHealthcare consists of four (4) major modules namely Admin module, Patient module, Doctor module and Pharmacist module. Each of these modules can perform the following routines:

1) Doctor:

- Can search for a particular patient.

- Can view the patient's complain.

- Can view scheduled appointments.

- Can cancel scheduled appointment.

- Can write the patient's medical reports.

- Can view the patient's radiology reports.

- Can view the patient's laboratory reports.

- Can view the prescription.

2) Patient:

- Can view his/her medical reports.

- Can post his/her complain.

- Can schedule appointment.

- Can view his/her radiology reports.

- Can view his/her laboratory reports.

- Can view his/her pharmacy information.

- Can ask for the prescription to get ready.

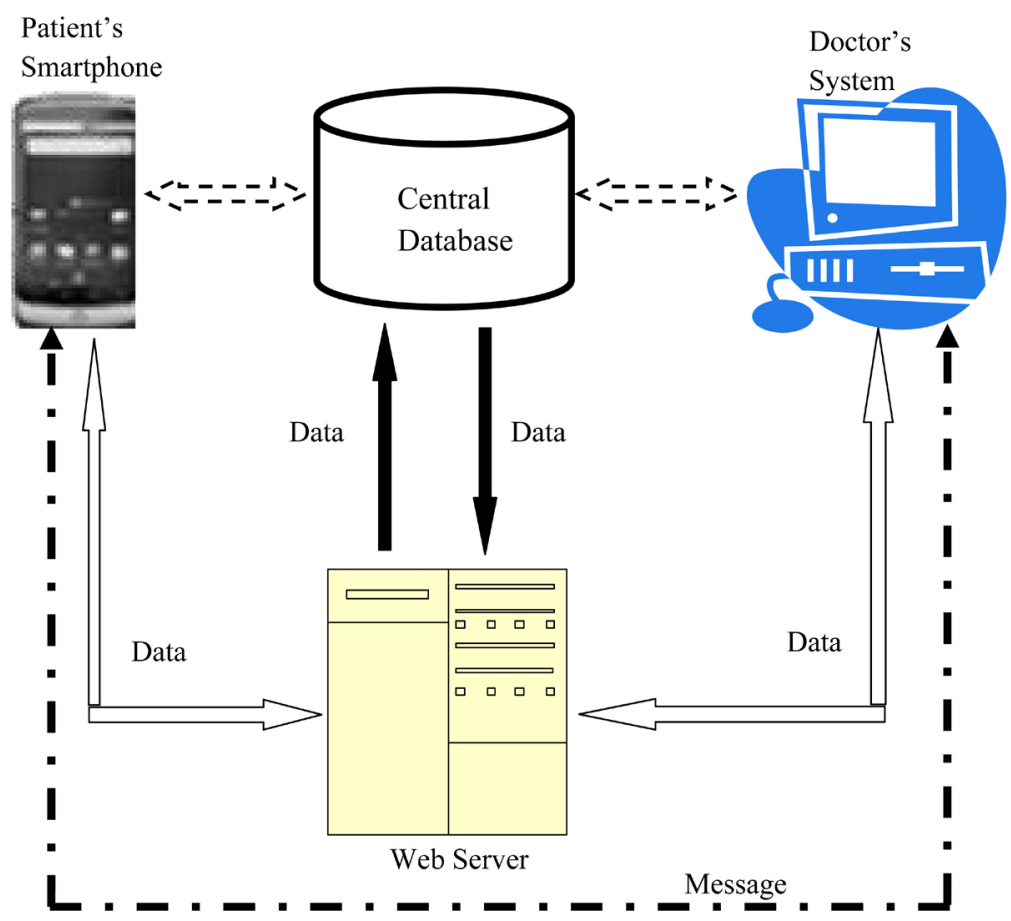

Figure 1. The proposed system architecture. 
3) Pharmacist:

- Can search for a patient.

- Can view the patient's doctor report.

- Can send an email to the patient after getting the prescription ready.

- Can view and update the information of the patient's insurance/payment status.

4) Administrator:

- Can search for a user.

- Can edit a user.

- Can check the retrieved update.

- Overall management of the system.

Some of the inputs to the system are as shown:

In Figure 2, the doctor logs in with his user name and password to access his account in the app but a new doctor must create an account to be able to log into the app.

In Figure 3, the patient will follow the same steps to gain access as the doctor.

Figure 4 shows one of the choices the patient can make after login into his page, which is to schedule an appointment with the doctor of his choice.

Figure 5 displays the dashboard where the doctor can book the patient for laboratory test and the type of test to be conducted.

In many cases, output is the main reason for developing the system and the basis on which the usefulness of the system is evaluated. In this work, the following are some of the outputs.

Figure 6 is the page that displays various information on the patient's profile.

Figure 7 shows the patient's medical report which can be accessed by both the doctor and patient.

Figure 8 is the page that displays the outcome of the laboratory test conducted on the patient.

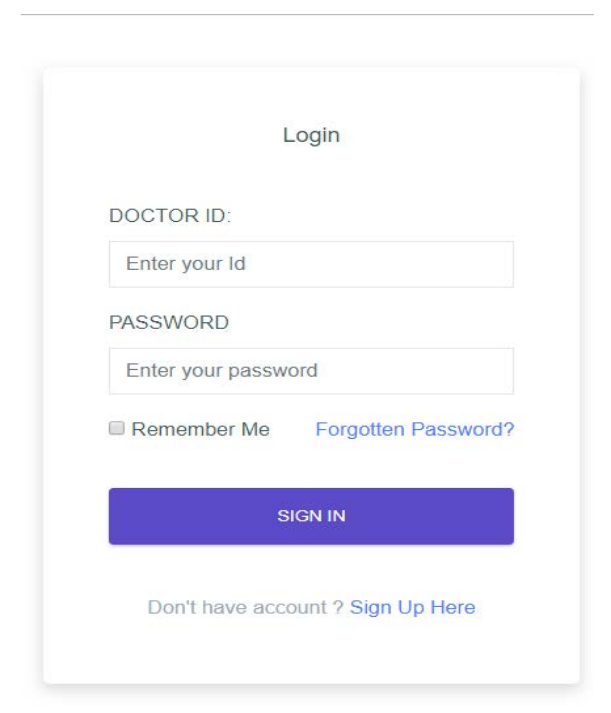

Figure 2. Doctor login input. 


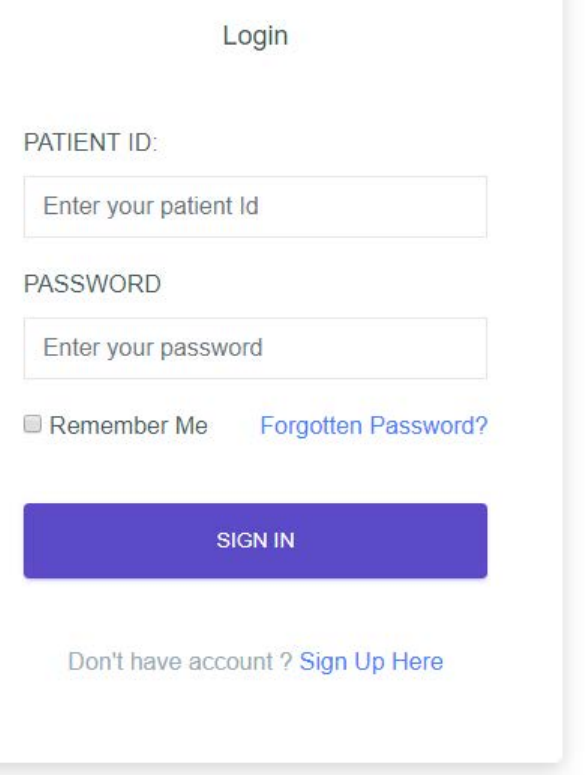

Figure 3. Patient login input.

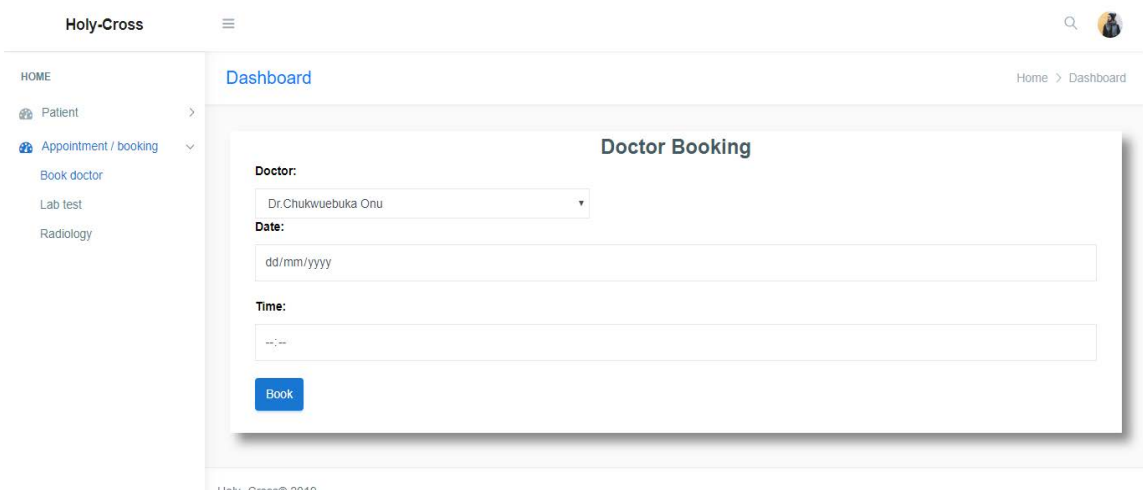

Figure 4. Doctor appointment booking input.

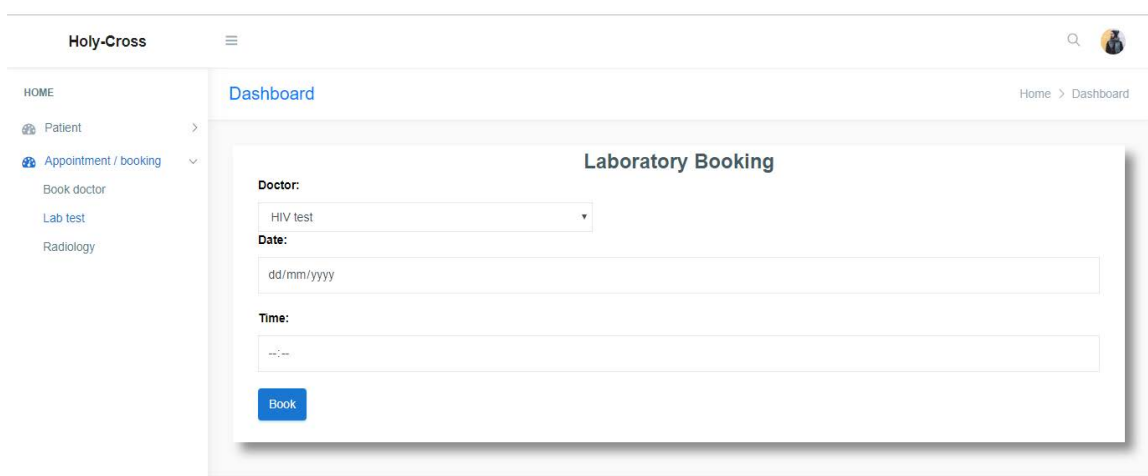

Hoy- CrossO 2019

Figure 5. Patient laboratory test booking input. 


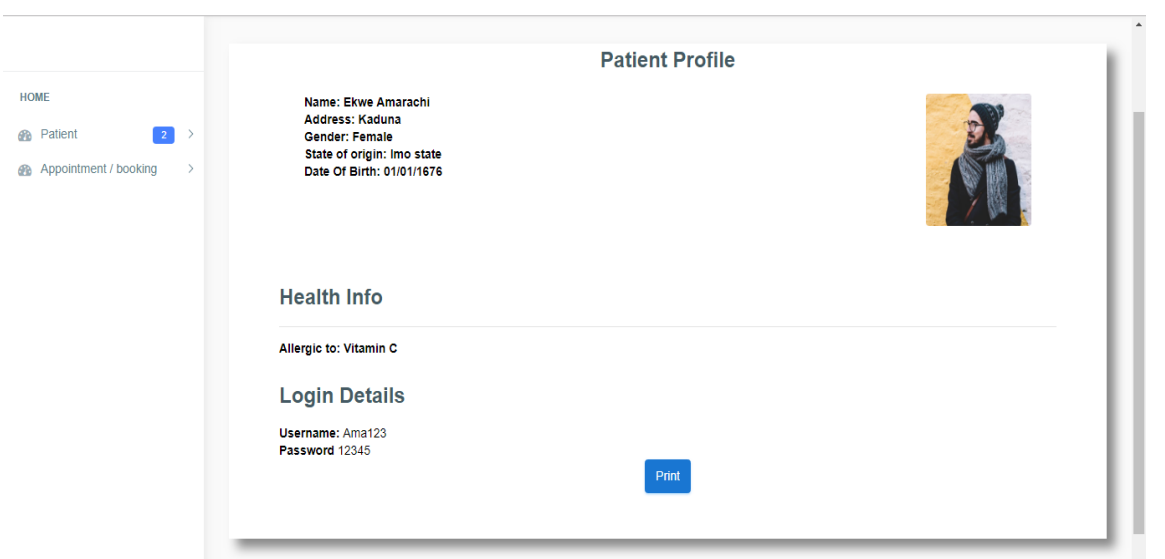

Figure 6. Patient profile output.

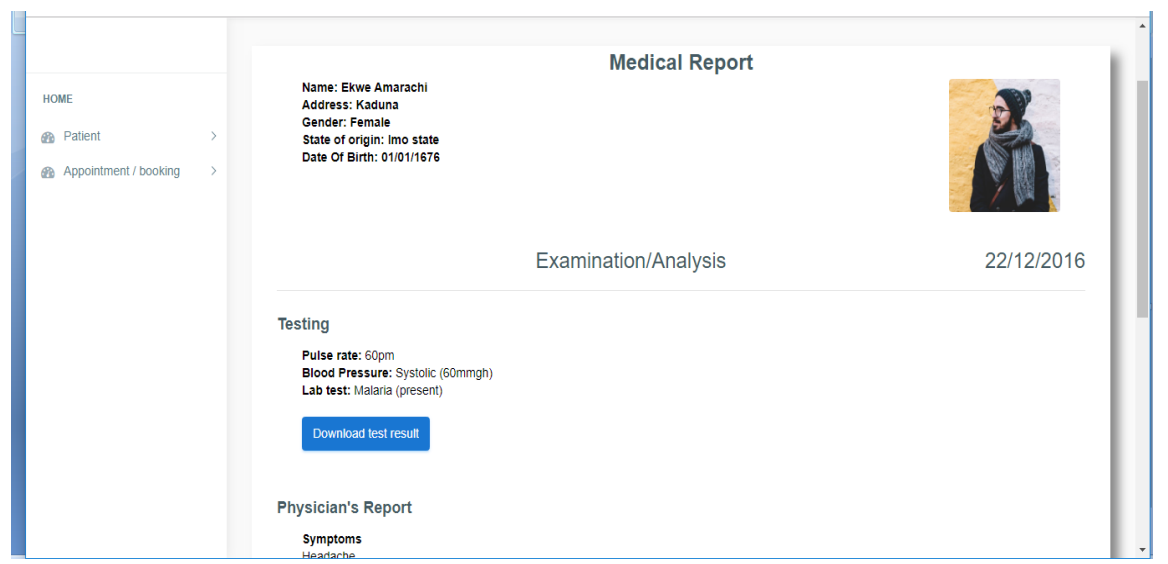

Figure 7. Patient medical report output.

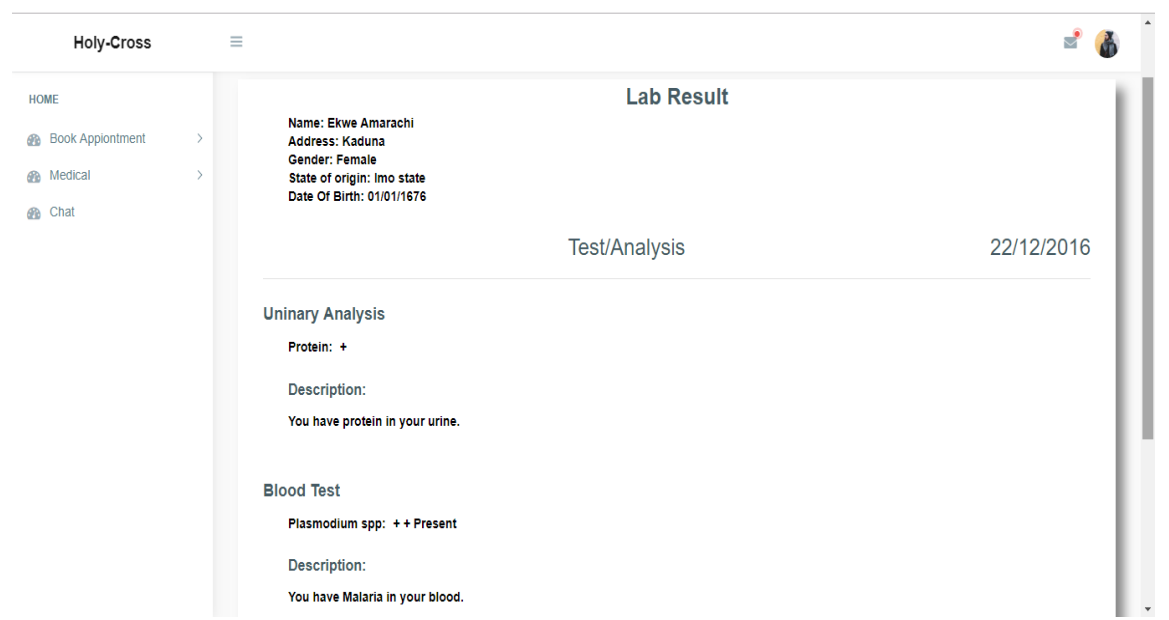

Figure 8. Patient laboratory result output.

\section{Module Description}

The coding phase deals with transforming the user requirements into a form that is implementable using programming language. The programming languages used to develop the front-end are JAVA and HTML while the back-end 
was powered with PHP and MYSQL. Different models were required to implement the entire design solution. There is innovation relationship among different modules. This includes which modules call other modules. The interface among different module identifies the exact data items exchanged among the modules.

\section{Doctor module:}

- The doctor shall register before login. He/She shall fill the registration form that consists of the following (First Name, Last Name, SIN, Specialization, Nationality, Gender, DOB, Address, Phone Number, Email, Position, User Name, Password) fields and receives an approval or denial via an email.

- The doctor shall be able to login with his/her user name and password; also he/she shall be able to logout.

- The doctor shall be able to search for a patient by entering patient's first name, last name and file number.

- The doctor shall be able to view scheduled appointments.

- The doctor shall be able to cancel appointments.

- The doctor shall be able to view the patient complain.

- The doctor shall be able to write report of his finding about the patient.

- The doctor shall be able to view patient's reports (medical report or laboratory report or radiology report).

- The doctor shall be able to add prescription and add it to the patient's file. Patient module:

- The patient shall register before login. He/she shall fill the registration form that consists of the following (First Name, Last Name, Nationality, SIN, Gender, DOB, Address, Phone Number, Email, User Name, Password) fields and receive an approval or denial via an email.

- The patient shall be able to login with his/her user name and password, also he/she shall be able to logout.

- Write and post his complain.

- The patient shall be able to schedule appointment.

- The patient shall be able to view his/her medical reports or laboratory reports or radiology reports.

- The patient shall be able to view his/her pharmacy information.

- The patient shall be able to ask for his/her prescription to get ready. Pharmacist module:

- The pharmacist shall register before login. He/she shall fill the registration form that consists of the following (First Name, Last Name, SIN, Gender, DOB, Address, Phone Number, Email, User Name, Password) fields and receive an approval or denial via an email.

- The pharmacist shall be able to login with his/her user name and password, also s/he shall be able to logout.

- The pharmacist shall be able to search for a patient by entering the patient's first name, last name and file number.

- The pharmacist shall be able to send an email to the patient after getting the 
prescription ready.

- The pharmacist shall be able to view and update the information of the patient's payment status.

\section{Administrator module:}

- The admin shall be able to login with his user name and password; and he shall be able to logout.

- The admin shall be able to search for a user (patient, doctor, nurse, and pharmacist) by user name, SIN and DOB to view the user's information.

- The admin shall be able to edit the user account.

- The admin shall be able to check the retrieved update.

- The admin shall have overall management of the system.

\section{Conclusions}

The application works for the benefits of the society and provides an interactive interface between the patient and the doctor. This paper proposed a mobile healthcare application that provides both healthcare providers and patients access to accurate and up-to-date information with less time and effort as well as improved efficiency of the information flow. The main advantage of this application is that doctors will be provided with full history of their patients' health status and patients will hold their data wherever they go. The proposed system will also help Medical Doctors to speed up diagnosis and treatment of patients through the advice and interaction with the patient.

The system is portable and can easily be installed and used on any mobile phones supporting Android OS. The use of this system can result in a reduction in number of hours spent searching for doctors and contacting them at the time of need. It also provides an interface that is easy to understand by the users and greatly helps in adapting to the use of this system.

\section{Future Work}

This application can be improved in the future by adding the following functionalities:

- Supporting video calls to discuss the problems with doctors.

- Implement hardware for monitoring status of the patient.

- Patients reports can be stored in encrypted form.

- The system can also be enhanced by using voice recognition feature of the Android.

\section{Conflicts of Interest}

The authors declare no conflicts of interest regarding the publication of this paper.

\section{References}

[1] Salmon, S., Brnt, G., Marshall, D. and Bradley, A. (2000) Telemedicine Use in 
Twonurse-Led Injuries Units. Journal of Telemedicine and Telecare, 6, 43-45. https://doi.org/10.1258/1357633001934113

[2] Avancha, S., Baxi, A. and Kotz, D. (2012) Privacy in Mobile Technology for Personal Healthcare. ACM Computing Surveys, 45, Article No. 3. https://doi.org/10.1145/2379776.2379779

[3] Zafar, A. and Hurko, M. (2012) Patients Gain When Doctors' Notes Are Online Retrieved.

https://www.cbc.ca/news/health/patients-gain-when-doctors-notes-are\%20online

[4] Online Health Records (2011)

https://www.cbc.ca/news/canada/toronto/online-health-records-popular-with

[5] Reuters, T. (2015) Hospital Patients May Worry Less after Seeing Their Medical Records.

https://www.cbc.ca/news/health/hospital-patients-may-worry-less-after-seeing-their -medical-records

[6] World Health Organization (1993) Doctor-Patient Interaction and Communication. Geneva, Document WHO/MNH/PSF/93.11.

[7] Google Play (2015) https://play.google.com/ 